\title{
When Black and White make Green: the Surprising Interplay of Structure and Pigments
}

\author{
Tianqi Sai ${ }^{a}$, Bodo D. Wilts ${ }^{\mathrm{b}}$, Alba Sicher ${ }^{\mathrm{a}, \mathrm{c}}$, Ullrich Steiner ${ }^{\mathrm{b}}$, Frank Scheffold ${ }^{\mathrm{d}}$, and Eric R. Dufresne ${ }^{\star a}$
}

\begin{abstract}
The natural world is teeming with color, which originates either from the wavelength-dependent absorption of light by pigments or from scattering from nanoscale structures, or both. While the latter 'structural color' has been a topic of intense study in recent years, the most vibrant colors in nature involve contributions from both structure and pigment. The study of structure-pigment interactions in biological systems is currently in its infancy and could inspire more technological applications, such as sustainable, toxin-free pigments and more efficient light harvesting.
\end{abstract}

Keywords: Absorption $\cdot$ Photonics $\cdot$ Pigments $\cdot$ Scattering $\cdot$ Structural color

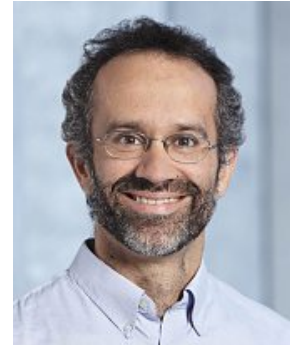

Eric Dufresne is the Professor of Soft and Living Materials at ETH Zürich. His current research concerns interfacial properties, mechanics, structural-color and self-assembly in biological and synthetic systems. Before moving to ETHZ, Eric was a graduate student at the University of Chicago, a postdoc at Harvard University, and a professor at Yale University. Eric is an Editorial Board member of Physical Review X.

Nature is excellent at manipulating light and producing color across the entire visible spectrum. Researchers have long been fascinated by nature's vibrant colors and the underlying mechanisms. ${ }^{[1]}$ Color production is typically classified as pigmentary or structural. Pigmentary color originates from wavelength-dependent absorption of light due to its interactions with the electronic structure of pigments and dyes. Structural color originates from the interaction of light with structures at much larger scales, comparable in size to the wavelengths of light. In recent years, there have been tremendous advances in our understanding of structural routes to color production, and researchers have designed a variety of artificial structures emulating the structural colors produced by nature. ${ }^{[2]}$ Yet, nature's most vibrant colors often defy this simple classification scheme, and emerge from the cooperation of scattering and absorption. ${ }^{[3]}$

Biological pigments can be roughly classified into two groups, broadband and wavelength-selective. Broadband pigments, like melanin, are ubiquitous and exist in bacteria, insects, animals, or humans, to just name a few. ${ }^{[4]}$ Eumelanin is the most commontype of melanin. It has strong absorption across UV and visible wavelengths, and usually appears in various shades of black or deep brown, depending on pigment concentration. Wavelengthselective pigments include chlorophylls in plants, carotenoids in plants and animals, and pterins in butterflies. ${ }^{[5]}$ Chlorophylls absorb light both in the red and in the blue regions of the visible light spectrum and appear as green. ${ }^{[6]}$ Carotenoids typically absorb wavelengths ranging from $400 \mathrm{~nm}$ to $550 \mathrm{~nm}$ and appear as red, orange, or yellow colors. ${ }^{[7]}$ Carotenoids are responsible for the colors of many plants and fruits, such as buttercups, carrots, corn and apricots. Animals can intake carotenoids by diet. The orange of goldfish and the yellow of the canary bird are classic examples.

Biological nanostructures producing structural colors are tremendously diverse, and are well reviewed in several recent articles. ${ }^{[8]}$ These photonic nanostructures are typically classified by the dimensionality and degree of translational order. The green and purple colors of Japanese jewel beetles are produced by a regular multi-layered structure, as shown in Fig. 1a,b. ${ }^{[9]}$ Different colors here arise from a simple tuning of the layer thicknesses. The diamond weevil produces color by scattering from a structure with three-dimensional long-range order (Fig. 1c-e). ${ }^{[10]}$ The eastern bluebird produces blue from a network with short-ranged order in three dimensions (Fig. $1 \mathrm{f}, \mathrm{g}$ ). ${ }^{[11]}$ Despite this wide range of morphologies, the dominant mechanism of color production in all of them is interference of light scattered from spatial gradients in the refractive index. ${ }^{[12]}$

Pigments and structure rarely work alone. Nature's vivid colors typically result from a finely tuned interplay between structural and pigmentary color. ${ }^{[13]}$ Here, our intuitions about how colors mix can be of little use. Black and white materials do not mix to make gray, but blue, or green, as described below.

The surprising interplay of pigment and nanostructure is evident in an investigation of the blue jay, where the coloration of an albino jay feather was compared to a normal dark-blue jay feather. ${ }^{[4 c]}$ Albinism is the lack of pigment production by an animal. Although the nanoscale morphologies of the albino and normal feathers are identical, the albino's feathers are missing the jay's characteristic saturated blue. Instead, they are white, with a faint hint of blue, as shown in Fig. 2a,b. Thus, melanin is essential in making a saturated blue, even though it is a broadband absorbing pigment. Indeed, melanins are found in the spongy medullary tissue of bird feathers in close proximity to the keratin-air nano-

\footnotetext{
${ }^{*}$ Correspondence: Prof. E. R. Dufresne ${ }^{a}$

E-mail: eric.dufresne@mat.ethz.ch

aDepartment of Materials, ETH Zürich, Vladimir-Prelog-Weg 5, CH-8093 Zürich

${ }^{b}$ Adolphe Merkle Institute, University of Fribourg, Chemin des Verdiers 4, $\mathrm{CH}-1700$ Fribourg

'Empa, Swiss Federal Laboratories for Materials Science and Technology, Laboratory for Biomimetic Membranes and Textiles, Lerchenfeldstrasse 5, CH-9014 St. Gallen

adepartment of Physics, University of Fribourg, Chemin du Musée 3, CH-1700 Fribourg
} 


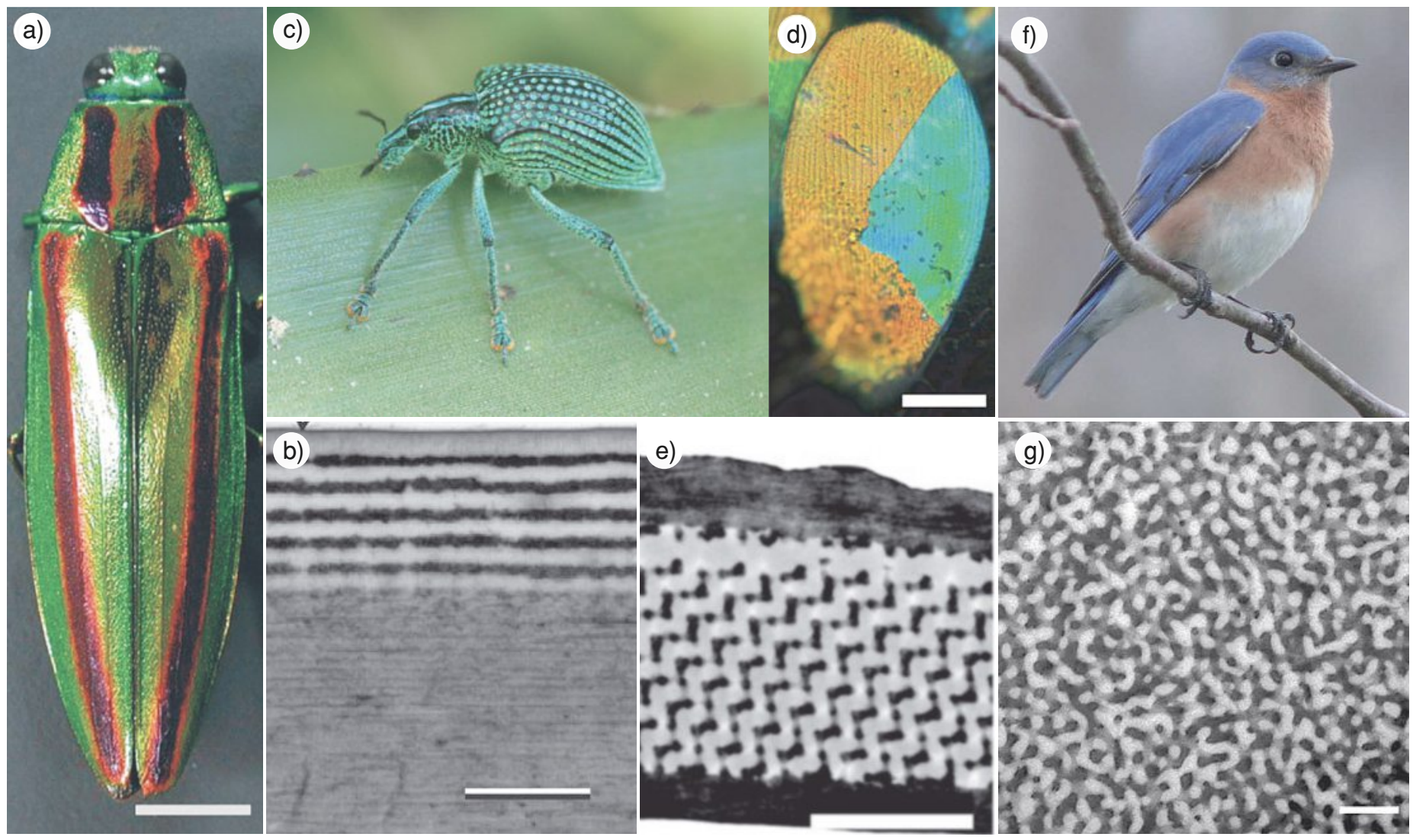

Fig. 1. Natural examples of structural color. a) Dorsal view of a female Japanese jewel beetle, Chrysochroa fulgidissima. The elytra and thorax are green with purple stripes (scale bar: $0.5 \mathrm{~cm}$ ). b) Transmission electron micrograph of purple sections of the jewel beetle elytra (scale bar: $1 \mathrm{~mm}$ ). c) The diamond weevil, Entimus imperialis, sits on a green leaf, with its black elytra studded with numerous yellow-green pits, sitting on a green leaf (Carlos M. Ribeiro). d) A single scale of the diamond weevil with a few differently colored domains (scale bar: $20 \mathrm{~mm}$ ). e) TEM of a single scale of the diamond weevil revealing a photonic crystal structure (scale bar: $2 \mathrm{~mm}$ ). f) Male Eastern Bluebird (Sialia sialis, Turdidae). g) Channel-type b-keratin and air nanostructure from back contour feather barbs of S. sialis. Fig. 1 a), b) reproduced with permission of Bodo D. Wilts. ${ }^{\left[{ }^{9]}\right.}$ Fig. $1 \mathrm{c}$ )-e) reproduced with permission of Royal Society. ${ }^{[10]}$ Fig. $\left.1 \mathrm{f}\right)$, g) reproduced with permission of Eric R. Dufresne. ${ }^{[11]}$

structures that define structural colors. ${ }^{[5 b]}$ Also, broadband pigments have been exploited to enhance coloration in a number of synthetic structurally colored materials. For example, Forster et. al. showed carbon black could enhance the non-iridescent green of isotropic packings of colorless polystyrene nanoparticles, as shown in Fig. 2c,d. ${ }^{[14]}$ With little carbon black, the films were white with a hint of green, Fig. 2c. With an excess of carbon black, they appeared black. However, when the concentration of carbon black was just right, a saturated green color appeared.

The basic physical principle of this surprising effect is rather simple. Even though the translational order of the nanostructure causes some wavelengths to be scattered more strongly than others, this preference is not perfect. Any photon will eventually be scattered if given multiple opportunities to interact with the nanostructure. Thus, thick samples tend to appear white. ${ }^{[15]}$ By introducing the right quantity of broadband absorbers to the material, only wavelengths that interact the most strongly with the nanostructure will be scattered from the sample before being absorbed.

Pigments need help from scattering, too. While pigments control absorption, we see most natural objects in reflection. Pigments only produce saturated colors in reflection when they are embedded in a strongly scattering, otherwise white, material. This important effect is familiar if you have ever bought paint. Once you have selected your color-of-choice, the clerk makes the paint by mixing the pigment with a strong white base. Nature uses the same trick. A nice example is the yellow feather barb of the American goldfinch (Carduelis tristis). Here, a carotenoid pigment is embedded in a nanostructure of colorless keratin and air.[16] When scattering from the nanostructure is suppressed by infiltration with an indexmatching substance, the vibrant color fades to a faint yellow.
In the examples above, scattering and absorption work independently, with distinct system components responsible for each function. While this simplifies the physics and leads to some simple design rules, many natural systems produce color by scattering from pigmented structures. For example, birds produce iridescent color by scattering from organized arrays of melanin-containing organelles. ${ }^{[5 b, 17]}$ Recently, this approach has been adopted in synthetic materials where polydopamine nanoparticles mimic melanosomes. While suspensions of these particles are black, they produce colors tunable across the visible spectrum when packed into arrays with short-ranged order or cast into thin films. ${ }^{[18]}$ Why are particles made from absorbing materials so effective at producing structural colors? While careful studies of the structure-property relations of these materials are lacking, some basic physical principles can provide some insights.

Scattering and absorption are intimately related, both deriving from the same material property: the dielectric function. The real part of the dielectric function determines the refractive index, and thus the scattering behavior. The imaginary part of the dielectric function determines absorption. The real and imaginary parts of the dielectric function cannot be varied independently, but must satisfy the Kramers-Kronig relation. ${ }^{[19]}$ For example, absorption over a narrow band of wavelengths results in a peak of the refractive index at longer, nearby wavelengths. This principle is nicely demonstrated by the common, brightly colored Pierid butterflies, which range in color from white to red. ${ }^{[20]}$ (Fig. 2e) Here, pterin pigments are concentrated inside rice-shaped beads within the butterfly wing scales. The pterins can play two roles, they (i) absorb light at short wavelengths and (ii) enhance light scattering at longer wavelengths. As shown in Fig. 2f, g, the orange wing scales 

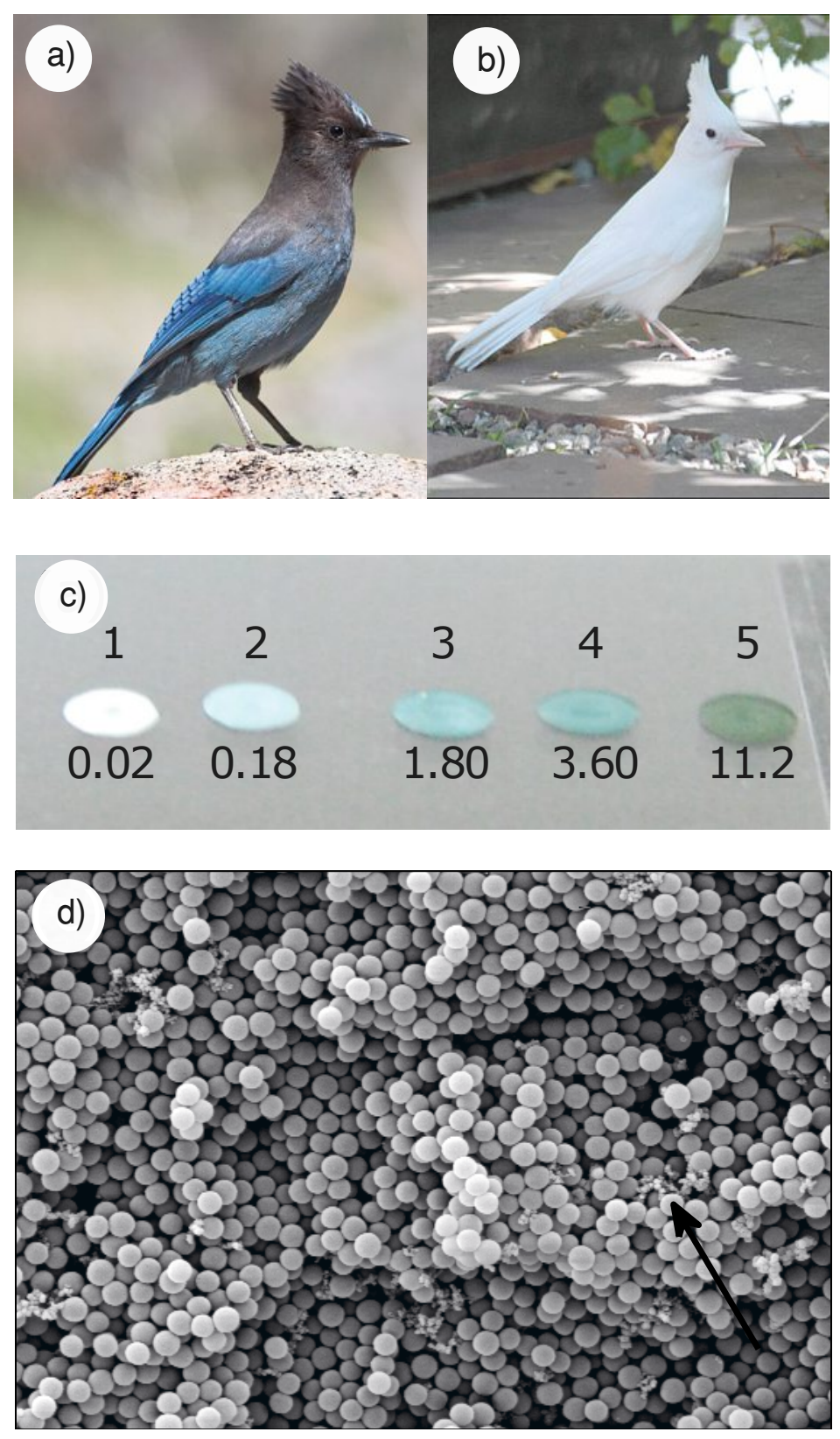

e)
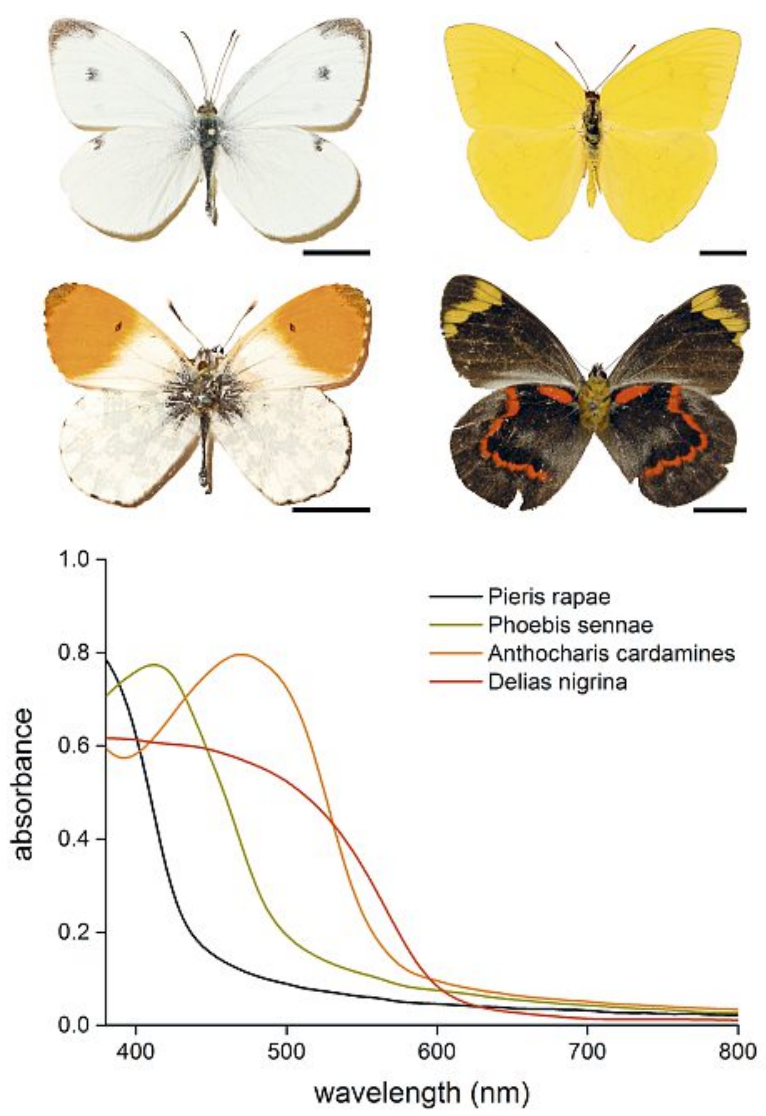

g)

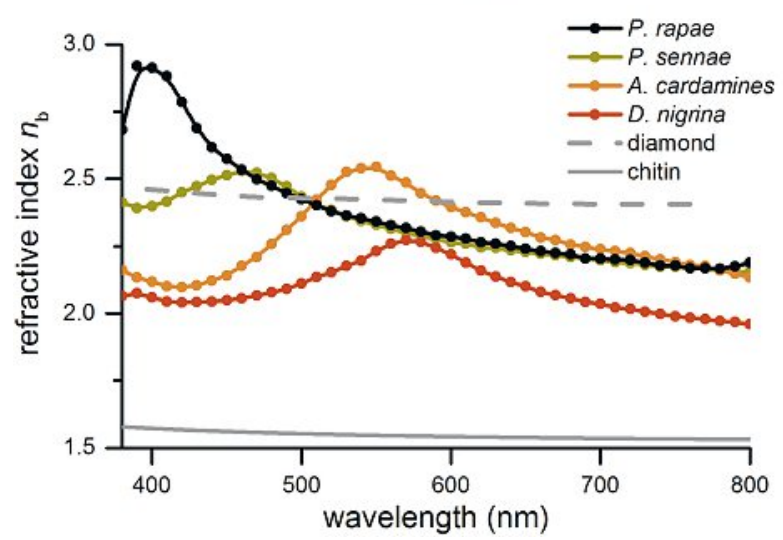

Fig. 2. Absorption enhances scattering. a) Photograph of Steller's jay by Stephen Ting. b) Photograph of the amelanotic Steller's jay by Bill Schmoker. c) Photograph of five drop-cast films of 226 and $265 \mathrm{~nm}$ PS spheres containing carbon black. Sample numbers and [CB] in wt\% appear above and below the samples, respectively. d) SEM image of the interior region of sample 3; the field of view is $7.8 \mu \mathrm{m}$ wide; a piece of CB is indicated with an arrow. e) Photographs of male pierid butterflies. Pieris rapae (top left). Phoebis sennae (top right). Anthocharis cardamines (bottom left). Delias nigrina (bottom right). f) Absorption spectra of pigmented beads of the four pierid butterflies. g) Real part of the refractive indices of pigmented beads. For comparison, the refractive index of chitin is added. Fig. 2 c), d) reproduced with permission of Jason D. Forster. ${ }^{[14]}$ Fig. 2 e)-g) reproduced with permission of Bodo D. Wilts. ${ }^{[20]}$

of Antocharis cardamines have an absorption peak absorption at $470 \mathrm{~nm}$, which leads to refractive indices above 2.2 in a band of wavelengths near $550 \mathrm{~nm}$. More generally, the enhancement of scattering by absorption also plays an important role in broadband absorbers, like melanin. In these cases, the monotonic decay of absorption with increasing wavelength results in high indices of refraction across the visible range and strongly enhanced scattering. [21]

These recent studies suggest that there are many exciting opportunities in basic and applied sciences for the study of structure-pigment interactions. The Kramers-Kronig relation offers a useful first step toward understanding these effects. However, the multi-scale nature of these optical composites raises fundamental questions about how the dielectric functions of base materials couples with scattering at the nanoscale to determine the effective dielectric function of the composite. We hypothesize that new phenomena could emerge when materials are designed so that the band of enhanced refractive index due to absorption overlaps with the band of wavelengths selected by the nanostructure. ${ }^{[13]}$

New insights into the interplay of scattering and absorption have the potential to impact technology on at least two fronts. First, commercial pigments rely on rare-earth elements or toxic chemical compositions and therefore can be expensive and dangerous to the environment. In fact, it is reported that wastewater from the textile factories is classified as one of the most polluting of all industrial sectors. ${ }^{[22]}$ Dyes can remain in water for decades, causing damage to the aquatic environment and limiting downstream beneficial uses of water, such as being used as drinking water. Optimized structure-pigment interactions could enable the use of more sustainable, non-toxic pigments and processes, as well as reduce the necessary amount of existing pigments needed to make the vibrant colors required for cosmetics, arts, and food. 
The interplay of scattering and absorption could also contribute to energy harvesting. ${ }^{[23]}$ In this domain, nature again has much to teach us. For example, the green leaves of plants focus and scatter light through thin layers of tissues to enable remarkably efficient light harvesting. ${ }^{[24]}$ Indeed, recent theoretical work has shown that locally ordered but globally disordered structures enhance the absorption of light for the same amount of material. ${ }^{[25]}$ New insights into the interactions of light scattering and absorption could not only lead to a better understanding of leaf physiology, but may also suggest new strategies for the design of light-harvesting devices, e.g. their use in solar cells or strongly absorbing materials. ${ }^{[26]}$

\section{Acknowledgments}

We thank Craig Brodersen for useful conversations. This research was partly supported through the National Centre of Competence in Research 'Bio-Inspired Materials', the Adolphe Merkle Foundation (to BDW and US), the Ambizione programme of the Swiss National Science Foundation SNSF (168223 to BDW).

\section{Competing Interests}

The authors declare that they have no competing interests.

Received: November 13, 2018

[1] a) L. Rayleigh, Proc. Roy. Soc. Lond. A 1917, 93, 565, DOI: 10.1098/ rspa.1917.0040; b) C. Raman, 'The origin of the colours in the plumage of birds', in Proceedings of the Indian Academy of Sciences, Section A, Vol. 1, Springer, 1934, pp 1-7; c) R. Hooke, 'Micrographia', Allestry, 1961; d) I. Newton, 'Opticks, or, a treatise of the reflections, refractions, inflections \& colours of light', Courier Corporation, 1979; e) B. Jackson, 'Kingfisher Blue: Treasures of an Ancient Chinese Art', Ten Speed Press, 2001; f) D. G. Stavenga, J. Tinbergen, H. L. Leertouwer, B. D. Wilts, J. Exp. Biol. 2011, 214, 3960, DOI: 10.1242/jeb.062620.

[2] a) M. Kolle, S. Lee, Adv. Mater. 2018, 30, 1702669, DOI: 10.1002/ adma.201702669; b) Y. Zhao, Z. Xie, H. Gu, C. Zhu, Z. Gu, Chem. Soc. Rev. 2012, 41, 3297, DOI: $10.1039 / \mathrm{c} 2 \mathrm{cs} 15267 \mathrm{c}$.

[3] D. G. Stavenga, Mater. Today: Proc. 2014, 1, 109, DOI: 10.1016/j.matpr.2014.09.007.

[4] a) B.-K. Hsiung, T. A. Blackledge, M. D. Shawkey, J. Exp. Biol. 2015, 218 3632, DOI: 10.1242/jeb.128801; b) P. A. Riley, Int. J. Biochem. Cell Biol. 1997, 29, 1235; c) M. D. Shawkey, G. E. Hill, J. Exp. Biol. 2006, 209, 1245, DOI: $10.1242 /$ jeb.02115.

[5] a) M. D. Shawkey, N. I. Morehouse, P. Vukusic, J. Roy. Soc. Interf. 2009, 6, S221, DOI: 10.1098/rsif.2008.0459.focus; b) G. E. Hill, 'Bird coloration: mechanisms and measurements', Harvard University Press, 2006; c) D. W. Lee, 'Nature's palette : the science of plant color', University of Chicago Press, Chicago, 2007.

[6] P. Lasareff, Biochem. Z. 1927, 182, 131

[7] a) O. Isler, 'Carotenoids', Vol. Band 23, Basel, Birkhäuser, 1971; b) R. O. Prum, A. M. LaFountain, C. J. Berg, M. J. Tauber, H. A. Frank, J. Comp. Physiol. B 2014, 184, 651, DOI: 10.1007/s00360-014-0816-1; c) K. Simpson, A. Cerda, C. Stange, Subcell Biochem. 2016, 79, 199, DOI: 10.1007/978-3-319-39126-7_7.

[8] a) P. Andrew Richard, J. Opt. A 2000, 2, R15; b) S. Kinoshita, S. Yoshioka, J. Miyazaki, Rep. Prog. Phys. 2008, 71, DOI: 10.1088/0034-4885/71/7/076401; c) S. Vignolini, E. Moyroud, B. J. Glover, U. Steiner, J. Roy. Soc. Interf. 2013, 10, DOI: 10.1098/rsif.2013.0394; d) P. Vukusic, J. R. Sambles, Nature
2003, 424, 852, DOI: 10.1038/nature01941; e) S. Yoshioka, S. Kinoshita, Proc. Roy. Soc. B 2006, 273, 129, DOI: 10.1098/rspb.2005.3314.

[9] D. G. Stavenga, B. D. Wilts, H. L. Leertouwer, T. Hariyama, Philos. Trans. Roy. Soc. B 2011, 366, 709, DOI: 10.1098/rstb.2010.0197.

[10] B. D. Wilts, K. Michielsen, J. Kuipers, H. De Raedt, D. G. Stavenga, Proc. Roy. Soc. B 2012, 279, 2524, DOI: 10.1098/rspb.2011.2651.

[11] E. R. Dufresne, H. Noh, V. Saranathan, S. G. J. Mochrie, H. Cao, R. O. Prum, Soft Matter 2009, 5, DOI: 10.1039/b902775k.

[12] S. Kinoshita, S. Yoshioka, Chemphyschem 2005, 6, 1442, DOI: 10.1002/ cphc. 200500007

[13] B. D. Wilts, A. Matsushita, K. Arikawa, D. G. Stavenga, J. Roy. Soc. Interf. 2015, 12, DOI: $10.1098 /$ rsif.2015.0717.

[14] J. D. Forster, H. Noh, S. F. Liew, V. Saranathan, C. F. Schreck, L. Yang, J. G. Park, R. O. Prum, S. G. J. Mochrie, C. S. O’Hern, H. Cao, E. R. Dufresne, Adv. Mater. 2010, 22, 2939, DOI: 10.1002/adma.200903693.

[15] a) D. S. Wiersma, Nat. Photon. 2013, 7, 188, DOI: 10.1038/Nphoton.2013.29; b) B. D. Wilts, X. Sheng, M. Holler, A. Diaz, M. Guizar-Sicairos, J. Raabe, R. Hoppe, S. H. Liu, R. Langford, O. D. Onelli, D. Chen, S. Torquato, U. Steiner, C. G. Schroer, S. Vignolini, A. Sepe, Adv. Mater. 2018, 30, e1702057, DOI: 10.1002/adma.201702057.

[16] M. D. Shawkey, G. E. Hill, Biol. Lett. UK 2005, 1, 121, DOI: 10.1098/ rsbl.2004.0289.

[17] a) C. M. Eliason, P. P. Bitton, M. D. Shawkey, Proc. Roy. Soc. B 2013 280, DOI: 10.1098/rspb.2013.1505; b) H. Durrer, 'Schillerfarben der Vogelfeder als Evolutionsproblem: elektronenmikroskopische Untersuchung der Schillerstrukturen, ihrer Morphogenese und Analyse von Selektionsmechanismen (speziell dargelegt am Beispiel der Hühnervögel)', Fretz, 1977.

[18] a) C. H. Lim, H. Kang, S. H. Kim, Langmuir 2014, 30, 8350, DOI: 10.1021/ la502157p; b) X. M. Yang, D. T. Ge, G. X. Wu, Z. W. Liao, S. Yang, Acs Appl. Mater. Inter. 2016, 8, 16289, DOI: 10.1021/acsami.6b03739; c) A. Kawamura, M. Kohri, G. Morimoto, Y. Nannichi, T. Taniguchi, K. Kishikawa, Sci. Rep. 2016, 6, DOI: 10.1038/srep33984; d) M. Xiao, Y. W. Li, M. C. Allen, D. D. Deheyn, X. J. Yue, J. Z. Zhao, N. C. Gianneschi, M. D. Shawkey, A. Dhinojwala, Acs Nano 2015, 9, 5454, DOI: 10.1021/ acsnano.5b01298.

[19] V. Lucarini, J. J. Saarinen, K. E. Peiponen, E. M. Vartiainen, 'Kramers-Kronig Relations in Optical Materials Research', Springer Berlin Heidelberg, 2006.

[20] B. D. Wilts, B. Wijnen, H. L. Leertouwer, U. Steiner, D. G. Stavenga, $A d v$ Opt. Mater. 2017, 5, DOI: 10.1002/adom.201600879.

[21] B. D. Wilts, K. Michielsen, H. De Raedt, D. G. Stavenga, Proc. Natl. Acad. Sci. USA 2014, 111, 4363, DOI: 10.1073/pnas.1323611111.

[22] A. B. dos Santos, F. J. Cervantes, J. B. van Lier, Bioresour. Technol. 2007, 98, 2369, DOI: 10.1016/j.biortech.2006.11.013.

[23] E. E. Narimanov, A. V. Kildishev, Appl. Phys. Lett. 2009, 95, DOI: Artn 04110610.1063/1.3184594.

[24] a) Q. T. Ho, H. N. Berghuijs, R. Watte, P. Verboven, E. Herremans, X. Yin, M. A. Retta, B. Aernouts, W. Saeys, L. Helfen, G. D. Farquhar, P. C. Struik, B. M. Nicolai, Plant Cell Environ. 2016, 39, 50, DOI: 10.1111/pce.12590; b) T. C. Vogelmann, J. N. Nishio, W. K. Smith, Trends Plant Sci. 1996, 1, 65, DOI: Doi 10.1016/S1360-1385(96)80031-8.

[25] F. Bigourdan, R. Pierrat, R. Carminati, arXiv preprint arXiv:1806.08188 2018.

[26] a) F. Pratesi, M. Burresi, F. Riboli, K. Vynck, D. S. Wiersma, Opt. Exp. 2013, 21, A460, DOI: 10.1364/Oe.21.00a460; b) S. Guldin, S. Huttner, M. Kolle, M. E. Welland, P. Muller-Buschbaum, R. H. Friend, U. Steiner, N. Tetreault, Nano Lett. 2010, 10, 2303, DOI: 10.1021/n1904017t; c) R. Schmager, B. Fritz, R. Hünig, K. Ding, U. Lemmer, B. S. Richards, G. Gomard, U. W. Paetzold, ACS Photon. 2017, 4, 2687, DOI: 10.1021/acsphotonics.7b01153; d) K. Vynck, M. Burresi, F. Riboli, D. S. Wiersma, Nat. Mater. 2012, 11, 1017, DOI: $10.1038 / \mathrm{Nmat} 3442$. 